\title{
The rheumatoid foot: a sideways look
}

\author{
Eric G Anderson
}

As with many other aspects of the foot, the rheumatoid foot, historically, has been treated symptomatically. If there is a painful tenosynovitis, synovectomy; a painful joint not responding to drug treatment, fusion; bony prominences (including metatarsal heads), excision; if the structure collapses, a valgus 'insole'; and if all else fails, bespoke footwear and discharge.

But is this good enough for 1990 when our knowledge of orthopaedic biomechanics has developed to a degree unthought of 20 years ago, our surgical expertise has been refined to an extent that was not possible 40 years ago, and the consequences of our treatment are appreciated as they could not have been when rheumatoid arthritis was first described? The answer must surely be an emphatic 'No'.

Before considering our approach to the orthopaedic management of foot disease we must firmly establish our objectives. We must consider the foot as a mechanism, both in its functional anatomy and pathology, for unless we understand what the disease process does to the functioning of the foot our management plan is liable to be misdirected and the gain to the patient less than optimum.

Of course, the foot does not function in isolation. It is at the end of a kinetic chain and at the interface between ground and body. Its relation to the more proximal joints must always be at the forefront of one's thinking (fig 1).

The possibilities and limitations of surgery must be defined, the contributions of orthotics and footwear explored, and then, and only then, can the place of these modalities be evaluated as an integrated treatment plan for any one subject.

Table 1 Objectives in treatment

Maintenance of independence

Relief of pain Preservation of

Footwear provision Footwear provision
Chiropodical care

Department of Orthopaedic Surgery, Western Infirmary Glasgow G11 6NT

E G Anderson

\section{Objectives}

It is easy to look for local objectives in the foot: freedom from, or reduction in pain, for example. But feet are patients' most direct interface with their environment. With the common duplicity of joint dysfunction, continued function of the feet becomes a major factor, and thus maintenance of the independence of the patient must be a first priority. This, of course, does not imply pain free locomotion without the use of aids. The ideal is not always the best objective. Consideration must be given to the subject's particular needs (table 1).

Maintenance of independence implies that foot function is of paramount importance and that treatment, however well intentioned, does not compromise it. This sideways look will be much concerned with this aspect, rather than

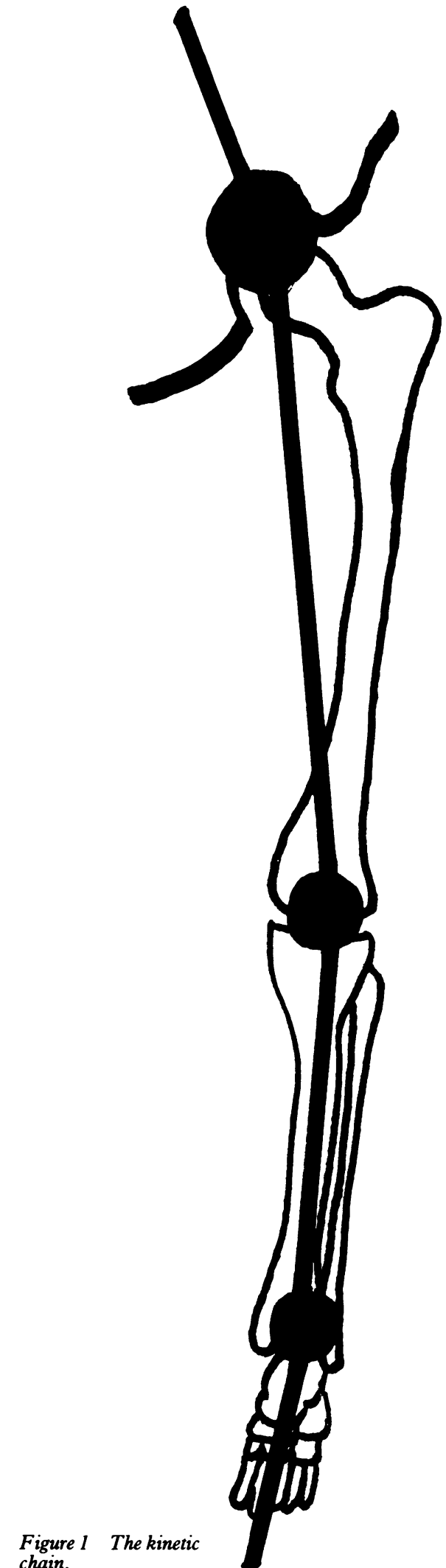

chain. 
delving into the niceties of the exact origins of pain in the rheumatoid foot. Pain, after all, is a subjective phenomenon, and its alleviation may require more than a surgeon or orthotist can offer. Nevertheless, the relief of pain or the attempted relief of pain, must feature highly as an objective.

Last of our primary objectives is the protection of the foot. Within the shoe the rheumatoid foot, liable to complications of dysvascularity as well as deformity, needs chiropodical care of skin and of nails. How often these are forgotten. And how much unnecessary suffering they can cause.

These stated objectives also imply the ability of the patient to cope with footwear. However well the foot functions, however long it remains pain free, the independence of that patient also depends on his/her ability to find suitable footwear. Shape is not the only problem patients may have. Footwear can also be adapted to ease the gait pattern and other joints affected by the disease process.

\section{Functional anatomy}

Over the years much myth has been perpetuated in the anatomy textbooks, particularly about the structural function of the foot. Who was not brought up on functioning arches and the primary loading function of the first metatarsal head? This, despite much thoughtful and pioneering work-for example, by Wood Jones in the $1940 \mathrm{~s}^{1}$ and Hicks in the $1950 \mathrm{~s}^{2}$ Our own work, supporting that of Cavanagh, dispels the anatomists' concepts of forefoot loading with significant consequences for forefoot surgery. Space does not admit of a detailed treatise on this subject, but we shall highlight the more important features that directly influence management.

We now consider the basic foot structure in the way Wood Jones did as two segmented beams, each segment bound firmly to the next by strong plantar ligaments which allow flexion at the bone interfaces but prevent extension (fig 2 ). These beams cross at the subtaloid joint, a joint whose axis lies in an oblique plane at $42^{\circ}$ plus or minus to the horizontal and $15^{\circ}$ plus or minus medial to the saggital plane. The pluses and minuses are important: they indicate a wide range of normality and, like the ranges of actual normal joint movement in the foot, these joints vary more than almost any other joint in the body. The system is complicated by the presence of the midtarsal joints, usually working in combination, which permits rotation between the forefoot and hindfoot. This combination of joints works effectively as a torque transmitter whereby foot rotation results in lower leg rotation and, of course, vice versa. This has significant consequences in the diseased hindfoot.

The foot and its ligaments are intrinsically stable at rest; the muscles of the leg are largely inactive. ${ }^{34}$ They come into their own during gait and are indispensable in the maintenance of the structure of the foot in action. Activity does imply stress, but stress can be applied to the foot without activity. Obesity, fortunately not such a common problem in the rheumatoid patient, increases stress and must be tackled seriously when in the presence of foot symptomatology.

Arguably, the most important single muscle in maintaining dynamic foot stability is the posterior tibial muscle, particularly in the rheumatoid patient. Why should this be more important than the peroneal muscles? Simply because of the natural tendency of the stressed foot to collapse into hyperpronation (or valgus) when the tibialis posterior becomes attenuated or ruptures. Loss of peroneal function is not accompanied by such dramatic alteration in foot structure.

In the normal foot, when the forefoot is flat on the floor, the heel is straight and the subtaloid joint is in its neutral position, neither inverted nor everted. It is common to find normal variations whereby the forefoot lies inverted or everted to the transverse plane relative to the hindfoot owing to the disposition of the midfoot joint axes, but compensated for by available midfoot rotation or subtaloid movement, particularly eversion. It is clear, therefore, that loss of such compensatory movements in these joints, for whatever reason, may result in abnormal rotational stresses being set up in the midfoot and the hindfoot on weight bearing with the production of a typical symptomatology and clinical picture (fig 3).

The function of the toes has been the subject of discussion and investigation. ${ }^{5}$ They are primarily used as stabilisers when the foot weight bears on the forefoot, and not for propulsion, at least at normal walking speeds. Feet which tend to supinate need to maintain balance, and the lateral toes claw desperately
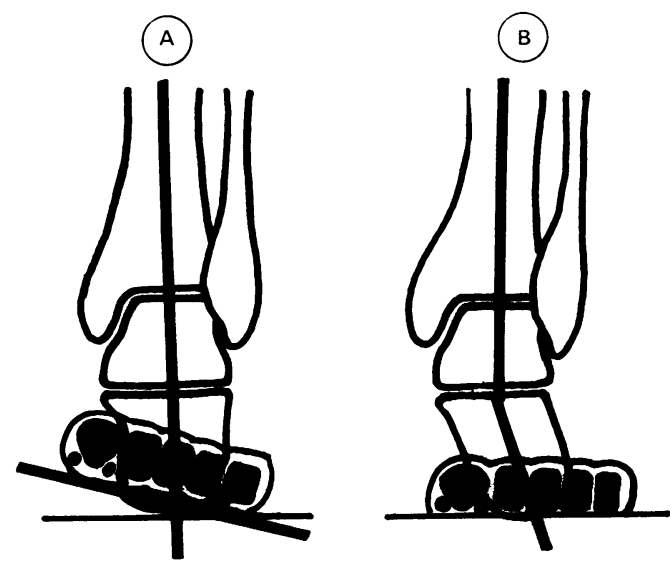

Figure 3 Inverted (varus) forefoot: (A) uncompensated (B) compensated. 
trying to maintain it. Pronating feet, on the other hand, find their forefeet and their toes taking up a relatively valgus posture, which forward motion can only accentuate, especially in the hallux. Thus hallux valgus is an inevitable consequence of posterior tibial division or rupture.

Normal gait consists not only of anteroposterior rocking of the foot but also of rotation. Three anteroposterior rockers are described: the body rolling over the heel as the calcaneum rolls inside the heel's skin like the wheel of a tank inside its track; the tibia rocking over the talus; and the metatarsal heads rolling over the plantar skin in a like fashion to the calcaneum. 'Roll over' is therefore a more appropriate description for this latter phase of gait than 'push off' (fig 4).

The high pressures seen under the hallux simply reflect the maintenance of body posture rather than any active propulsion into space. Although mention has been made of metatarsal head roll over and the pressures generated thereunder, it has been shown that in the normal population $48 \%$ have maximum forefoot pressure under the second and third metatarsal heads, $17 \%$ under the first head alone, $11 \%$ evenly under the first, second, and third heads, and $24 \%$ have a large lateral component of pressure under the fourth or fifth heads at least equal to the pressure under the first, second, or third heads (fig 5). ${ }^{6}$

To return to our normal gait pattern: on heel strike the foot usually hits the ground supinated, tends to pronate in stance phase, and supinate again on roll over. Clearly any inability of the leg to rotate will affect the ability of the foot to rotate within a functioning hindfoot complex, and contrariwise, a diseased hindfoot complex will affect the natural rotational movements of the foot or leg, or both. Thus the foot is inextricably linked to knee and hip function as part of the kinetic chain.

\section{Pathology}

Three basic pathological problems affect the foot:

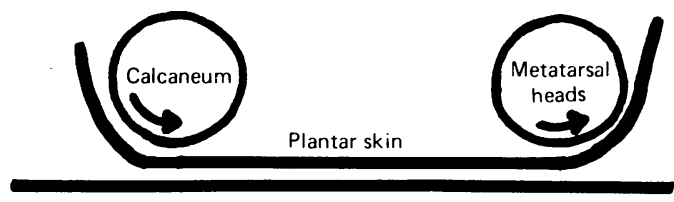

Figure 4 The plantar articulations.
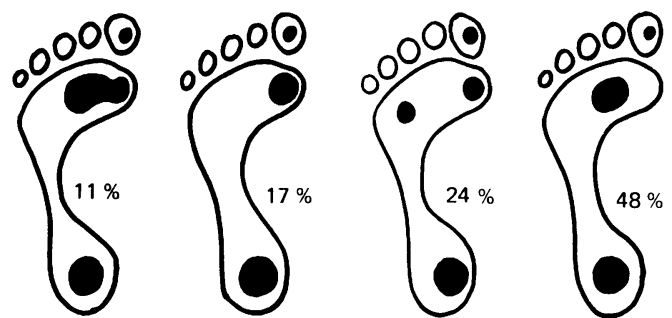

Figure 5 Distribution of pressure under normal feetnormal patterns.
1 Extrinsic disease elsewhere in the leg which alters the function of the foot. A clear example of this is the varus knee, which results in the foot hyperpronating to produce a foot flat in the stance phase of gait as well as the pronated position adopted by simple standing (fig 6). But there are knock on effects too. The subtaloid and midtarsal joints may be stressed at the extremes of their ranges, resulting in permanent alteration of the joint axes and secondary damage to the joint. Of more immediate importance, the posterior tibial tendon is put under immense strain and may attenuate or ultimately rupture.

2 Intrinsic disease-the joints. There is a tendency for either forefoot or hindfoot to be affected, unusually both. In the former the metatarsophalangeal joints bear the brunt, but in the latter the midtarsal, subtaloid, and ankle joints may all be involved initially with a synovitis before the degenerative changes develop. The talonavicular joint is often affected early, and synovitis of this joint should be looked for routinely.

3 Intrinsic disease-tendons and sheaths. Although any one of the tendons passing over or around the ankle may become diseased, the consequences are, as one might expect from the foregoing, much more severe for the foot if the posterior tibial tendon is affected. Early synovectomy is worthwhile to stem the disease progress in an attempt to reduce or delay tendon infiltration, incompetence, and ultimate rupture, with all the consequences this has for foot function. ${ }^{7}$

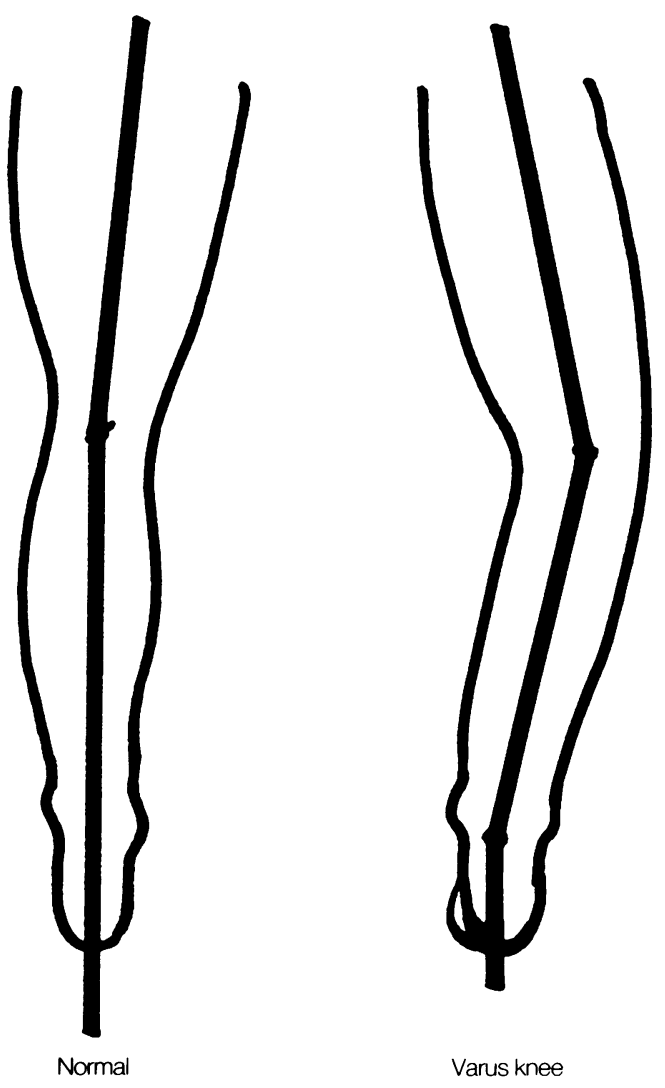

Figure 6 Effect of varus knee on foot alignment. 


\section{Examination and investigation}

From discussion so far it should be evident that examination should include the whole leg. Exposure to include the knee is mandatory; more if required. Examination must include active and passive movements-active motion against resistance being useful in determining the integrity of tendons. The function of the hindfoot can be shown by standing the patient on tiptoe and looking at the heels from the rear (Do they invert?) and the shin from the front (Does it rotate externally?). For those patients who cannot stand on tiptoe, passive dorsiflexion of the relaxed weightbearing hallux produces the same results.

Radiographs should always be taken weight bearing, and a lateral view should be routine. Specialised views, such as the weightbearing metatarsal profile, do not contribute much in this instance, as it is often impossible to position the patient satisfactorily on account of pain. As with any surgery in the rheumatoid patient the anaesthetist will appreciate radiographs of the cervical spine.

Much interest is now being shown in foot pressure measuring devices. The accuracy and repeatability of some is in doubt, and although many multicoloured pictures and a mass of data look impressive and can be readily accumulated, interpretation is difficult and much basic work remains to be done before this information can be interpreted with any validity. To date the dynamic pedobarograph with the Sheffield computerised analysis system is the most reliable. $^{8}$

More helpful are simple video gait studies, where the video picture can be analysed at a later date frame by frame. It is, however, time consuming and needs to be viewed by the clinician personally. Typed reports can be misleading and are next to useless. Photography

Table 2 Surgical options

\begin{tabular}{l}
\hline Bone \\
Osteotomy \\
Excision \\
Joint \\
Arthroplasty \\
Excision \\
Replacement \\
Realignment \\
Fusion \\
Tendon \\
Synovectomy \\
Transfer \\
Division \\
Repair \\
\hline
\end{tabular}

before operation is a neglected method of recording, and more consideration might be given to obtaining good clinical photographs at strategic points during treatment.

Routine blood examinations should not need further discussion.

\section{Treatment armamentarium} SURGERY

First in the list, only because it is a surgeon who writes. The options in surgery are limited and basic (table 2).

Osteotomy is helpful in altering the alignment of structures, particularly in situations of fixed deformity, but care must be exercised in order not to replace one problem with another.

Excision of part or all of one of the bones of the small toes still has a useful place. Amputation of all toes does not.

Joint replacement arthroplasty has been more successful in the ankle joint than in the foot itself; its use in the first metatarsophalangeal joint is debatable, and in the second to fifth even more so. Excision arthroplasty is still the mainstay of forefoot rheumatoid surgery in one form or another. ${ }^{9-11}$

Fusion is a much underused and underestimated procedure which deserves more atten- tion in rheumatoid disease. It has a significant part to play in the prevention of the development of structural deformity.

Tendon sheaths have been subjected to synovectomy for a long time in the history of the disease. Repair of the acute rupture of the posterior tibial tendon is a very worthwhile procedure, but too often it is seen too late for primary repair to be successful. Then, or if diseased beyond salvation, tendon transfer may give acceptable results. Tendon transfers also find a place in the treatment of the retracted small toe, giving a cosmetically acceptable, though functionally equally worthless, digit.

Simple tenotomy has little to offer, except perhaps in combination with other procedures.

\section{ORTHOTIC}

Orthoses are external appliances which influence the function of a part in one way or another. Three groups should be considered:

1 Orthodigital splinting with a silicone rubber putty, which can be moulded directly to the patient's toes to protect or correct mobile deformity. Latex protective covers also fall into this category (fig 7).

2 Foot orthoses. It seems rather obvious to state that all feet are different, hence there should be an insistence on all foot orthoses being made to foot casts. Much is possible now with new techniques of fabrication, new materials and, most importantly, new understanding of the biomechanical function of these orthoses (fig 8).

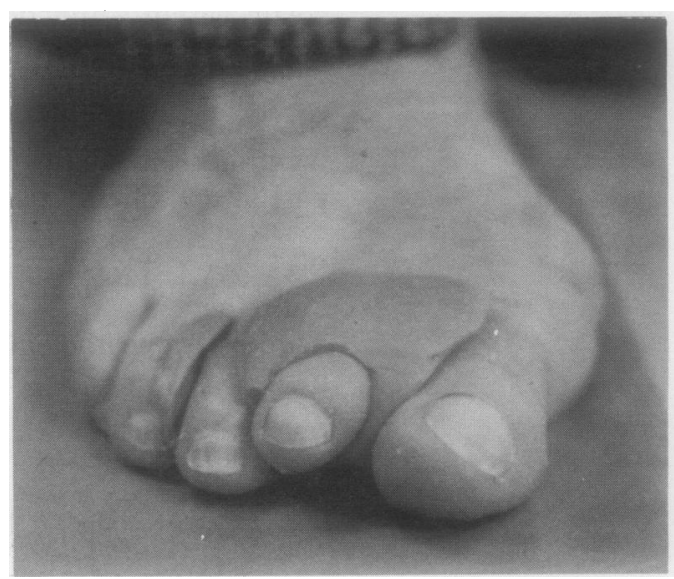

Figure 7 Orthodigital splint.

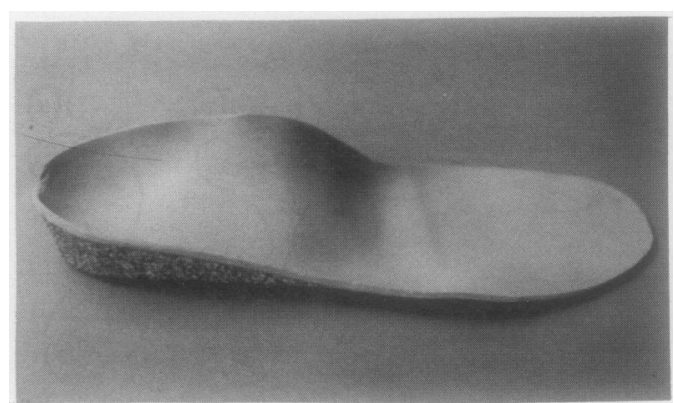

Figure 8 Functional casted foot orthosis. 
3 Leg orthoses. The ankle foot orthosis is most used, sometimes in conjunction with a separate foot orthosis. Although the 'drop foot splint' is the commonest in general orthopaedic practice, the rigid ankle foot orthosis is probably the most useful in rheumatoid disease.

\section{FOOTWEAR}

The requirements of rheumatoid patients are for footwear which is comfortable (no pressure, no rubbing), lightweight, durable, stylish, and, preferably, waterproof. This, of course, is an almost impossible collection of demands.

Patients' own footwear, as well as stock footwear, can have simple useful adaptations made to the soles and the heels that help their gait immeasurably.

\section{CHIROPODY}

An understanding and skilled chiropodist is an absolutely invaluable member of a caring team, just as is the orthotist and shoemaker. Regular attention to nails and callosities and orthodigital splinting all fall within his/her ambit.

\section{WALKING AIDS}

These must be included for completeness if for no other reason. Two feet may be insufficient on their own for safe ambulation and the assurance of a stick or other aid can mean the difference between independence and immobility through fear.

\section{Treatment principles}

Before discussing the methods of achieving treatment aims it is worth enumerating some basic principles:

1 Synovectomy of tendon sheaths should be performed at an early stage of disease as soon as there is evidence of a hypertrophic synovitis.

2 The weightbearing heel should be kept under the load line of the leg.

3 Mobile joints should be kept mobile wherever possible, unless their mobility is outside the normal range, allowing collapse of the foot architecture.

4 During gait the body load line should pass forward through the foot without producing abnormal rotational forces in the foot.

5 Foot movement should maintain the three rockers and if not this should be compensated for by adaptation of footwear.

6 Loss of forefoot to hindfoot alignment should be compensated orthotically.

7 Forefoot surgery should consider the problem of pain rather than function. By the time surgery is indicated it is destructive rather than constructive.

\section{Treatment}

Rheumatoid disease in the foot tends to present in one of three ways: talonavicular arthritis, peroneal or posterior tibial tenosynovitis, or as metatarsalgia. Although forefoot and hindfoot disease can coexist, commonly the patient is inflicted with one or the other.

HINDFOOT

The role of the posterior tibial tendon cannot be emphasised too strongly as it is the key to the most difficult to manage of all the rheumatoid foot problems-the collapsed pronated foot. Early synovectomy of the tendon sheath not only relieves discomfort but will possibly delay or prevent attenuation and rupture. Steroid infiltrations always run the risk of intratendinous injection, which may hasten the day of rupture.

When the diagnosis of ruptured posterior tibial tendon has been made, in the presence of a correctable hyperpronation, transfer of the flexor digitorum longus tendon to the distal posterior tibial tendon stump has been found to give acceptable results. ${ }^{712}$

Even with an affected posterior tibial tendon the midfoot may start to collapse owing to disease in these joints. Talonavicular joint disease is a not uncommon presenting form in the foot. Our experience with these feet, tending to collapse but still correctable, is to fuse the talonavicular joint to provide the foot with a stable, medial beam and prevent the calcaneum taking up a fixed valgus position. This needs to be carried out at the first signs of midfoot hyperpronation, and we routinely use cancellous iliac bone graft to ensure sound union.

Hyperpronation, by virtue of its mechanics, inevitably results in the hallux being forced into valgus even in the absence of rheumatoid disease in the first metatarsophalangeal joint. In this situation fusion of the first metatarsophalangeal joint is the preferred option. This does not apply when there is severe forefoot disease present.

Where the foot has collapsed into a pronated valgoid position, the requirements are multiple. The heel is no longer under the leg, the midfoot is twisted, often to the extent that the talar head starts to bear weight medially, and the gait becomes flat footed with the loss of the anterior rocker. A simple medial translational calcaneal osteotomy can help put the loadbearing area of the heel back under the loadbearing line of the leg (fig 9) and relieve much pain due to turning moments generated in the ankle/subtaloid complex on weight bearing.

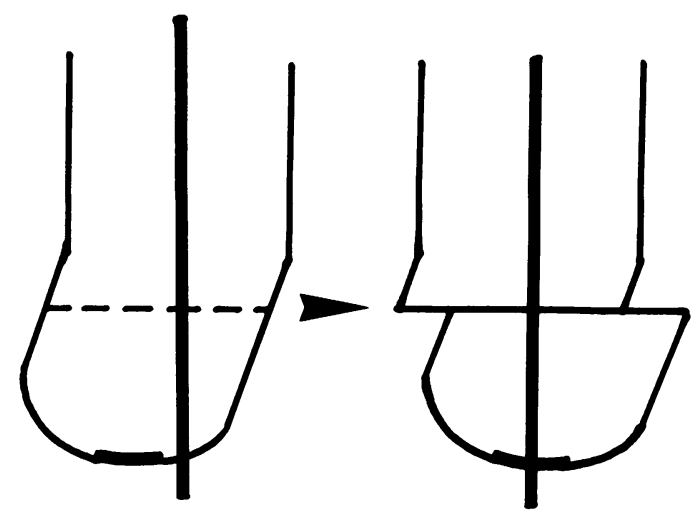

Figure 9 Translational calcaneal osteotomy. 


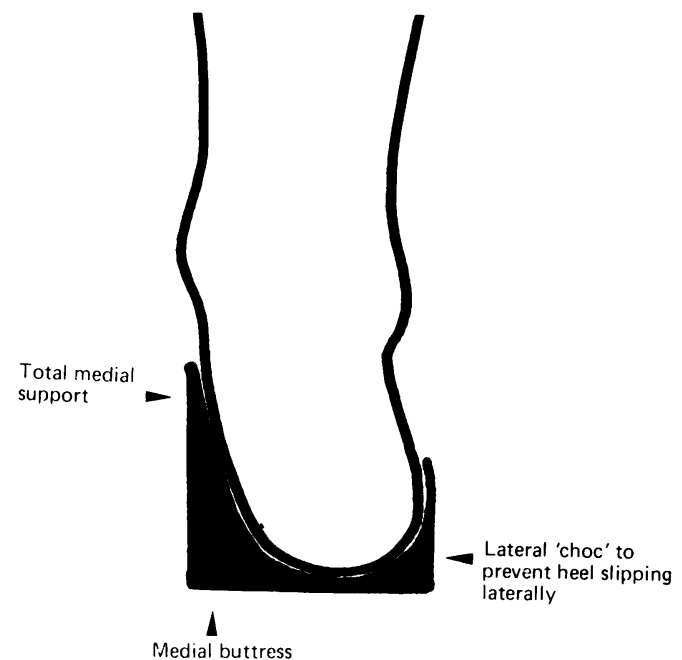

Figure 10 Orthotic support of the collapsed (valgus) foot.

The collapsed midfoot, however, is not amenable to simple surgery. Its accommodation within bespoke footwear is required but the 'overhanging' midfoot must be supported by an appropriate orthotic insole (fig 10). This inevitably leads to a bulky shoe, and all the skills of the shoemaker and orthotist are required to keep the weight down to manageable proportions for the patient.

Subtalar fusion is rarely indicated on these principles but if carried out the need to ensure accurate alignment of hindfoot and forefoot is essential.

\section{FOREFOOT}

With the forefoot intermetatarsal head pain is a common symptom. It has been suggested that rheumatoid disease of the intermetatarsal bursae is sufficient to cause either stretching or compression of the interdigital nerves. The symptoms have been relieved by infiltration of the bursae with steroid. We now recognise, however, that forefoot malalignment, in particular the inverted forefoot, can present in nonrheumatoid patients with similar symptoms, and there is no reason to expect that the rheumatoid foot is any different. Careful examination of the foot is, as always, essential and any malalignment with symptoms which does not have any other evidence of intermetatarsal bursitis or fails to respond to steroids should be compensated orthotically. Orthotic insoles for these patients need to be made with more pliable, shock-absorbing materials otherwise they will not be tolerated.

It is most uncommon for the full blown picture of the Morton's neuroma to appear, but if it does, resection of the traumatised segment of nerve may be necessary.

The occasional retracted toe can be best dealt with by excising the proximal 4/5ths of the proximal phalanx, by dividing the long extensor tendon at the level of the metatarsophalangeal joint, and by suturing the distal cut end to the long flexor tendon in the gap. The cosmetic effect is excellent, though functionally the toe remains equally useless (fig 11) (Stainsby, unpublished data).

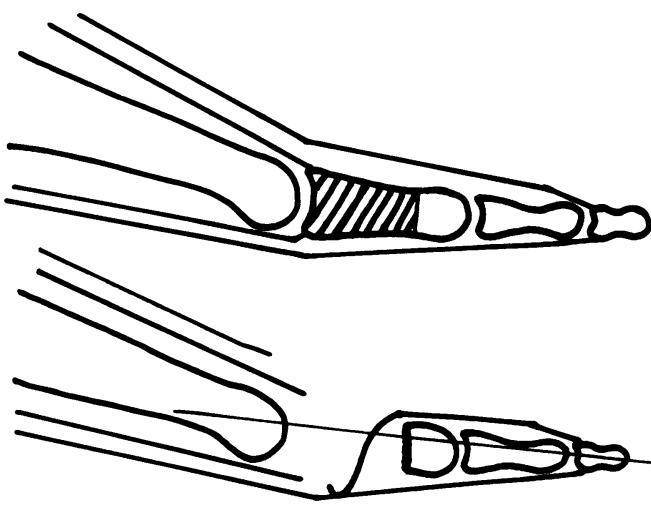

Figure 11 Proximal partial phalangectomy (after Stainsby).

When dealing with mallet toes it is probably worth remembering to tenotomise the flexor tendon even if distal interphalangeal joint fusion is being attempted.

Treatment of the destroyed metatarsophalangeal articulations with gross retraction of the toes has been a matter of debate. Stevens' review of forefoot excision arthroplasty versus footwear suggested that there was no difference in the comfort of the two groups after five years. ${ }^{13}$ This may be so, but the immediate relief to those patients after surgery is very gratifying, both for the patient and the surgeon, and we still consider it a worthwhile procedure. ${ }^{14}$

Our own preference is for the operation described by Kates, Kessel, and Kay in 1967. ${ }^{11}$ They advocated the excision of all metatarsal heads through an elliptical plantar incision, excising the skin overlying the metatarsal heads. The first metatarsal head can be excised through a medial longitudinal excision. Much has been made of the need to have the metatarsal stumps load bearing equally, and rightly so. An artistic curve on a non-weightbearing radiograph, taken on the theatre table does not necessarily equate with functional comfort. Our practice is at operation to push up against the metatarsal tread with the back of the hand, when any undue prominence can be easily felt and readily dealt with; perhaps still not very scientific, but a little more practical. What is not acceptable is excision of the second to fifth metatarsal heads combined with the Keller's procedure. The first metatarsal head is no more able to cope with the extra loading than any other prominent metatarsal head.

Treatment of the ankle is discussed by Kirkup (pp 837-44, this issue), but it is worth noting that the stiffened ankle, whether by disease, surgical fusion, or disappointing arthroplasty, reduces or eliminates the second rocker of the gait cycle. This can be compensated by a technique used by the prosthetists in their artificial SACH foot-solid ankle cushion heel. The incorporation of a spongy wedge into the heel of the shoe aids the forward propulsion of the body on heel strike and contributes significantly to an improved gait in these patients (fig 12). ${ }^{15}$

Over the years the advised indications for the Helal oblique metatarsal osteotomies have changed, but many have been disappointed 


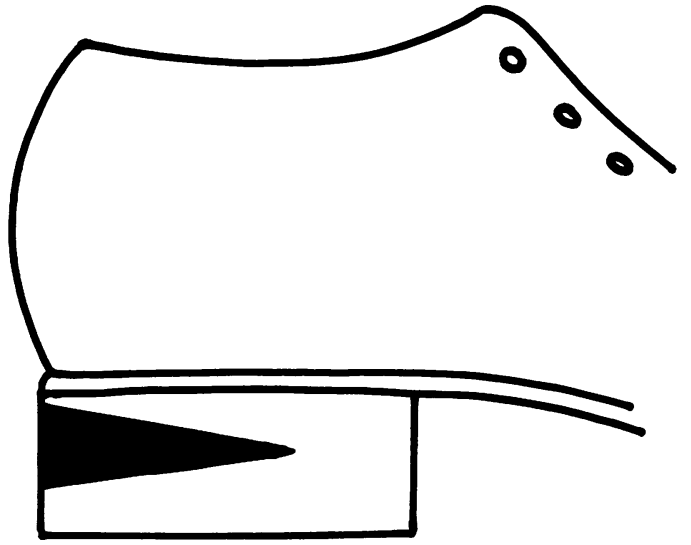

Figure 12 Cushion wedge heel.

with the results, particularly when used as originally described for midfoot metatarsalgia, and we have all but abandoned it. The biggest problems have been with high pressure transfer to the adjacent metatarsal heads after the operation, and to non-union of the osteotomies, although it should be said, the latter were rarely troublesome to the patient.

At an earlier stage, where the hallux is drifting into valgus, the foot pronating, but where the lesser metatarsophalangeal joints are not subluxed, fusion of the first metatarsophalangeal joint may be indicated. ${ }^{16}$ The loss of first metatarsophalangeal movement may be compensated with a rocker sole modification to the patient's footwear. The extra weight of this may be as disadvantageous as the loss of the forefoot rocker to the patient, but there is no certain way of predicting this.

Finally, the rheumatoid foot may also be a dysvascular foot, and this may limit the amount of surgery it is possible to carry out. It also results in atrophy of the fibro-fatty pads under the heel and the metatarsal heads that permit the first and third rockers to function painlessly. Under the forefoot the patient complains of 'walking on marbles' (compare the purely rheumatoid diseased forefoot described as 'walking on broken glass'). This tissue loss can only be partially compensated by the provision of an insole of one of the new shear-absorbing materials (PPT*, Spenco†). A word of caution about using too thick an insole of such materials as, although it may be more comfortable to stand on, it may create a degree of instability that impairs the patient's proprioceptive feedback mechanism-they do not know where their feet are.

Dysvascular toes need protection, and the use of orthodigital splints to prevent chaffing, even in bespoke footwear, should always be considered. Regular foot care is essential and the place of the chiropodist in the treatment of this type of foot cannot be overeinphasised.

\section{Epilogue}

The possible ways of management are endless and some commoner problems have been used to illustrate the particular principles of treatment and their use in fulfilling the general aims of patient care. The individual surgeon or doctor may find his way to different solutions; it matters not as long as these aims and principles are adhered to, for it is far easier in foot surgery to render a foot worse off than to cure it.

1 Jones F W. Structure and function as seen in the foot. London: Baillière Tindall, 1944.

2 Hicks J H. The mechanics of the foot. 1. The joints. $\mathcal{f}$ Anat 1953; 87: 345-57.

3 Basmajian J V, Stecko G. The role of muscles in the arch support of the foot. F Bone foint Surg [Am] 1963; 45: $1184-90$

4 Inman V T, Ralston H J, Todd F. Human walking. Baltimore: Williams and Wilkins, 1989

5 Hughes J, Gerber C, Jagoe R, Clark P, Klenerman L. The importance of toes in walking. Ann R Coll Surg Engl 1988; 70: 177.

6 Anderson E G, Harrison D. Foot pressure patterns in normal feet. Ann R Coll Surg Engl 1988; 70: 176-7.

7 Johnson K A. Tibialis posterior tendon rupture. Clin Orthop 1983; 177: 140-7.

8 Franks C I, Betts R P, Duckworth T. A microprocessor based image processing system for dynamic foot pressure studies. Fournal of Medical and Biological Engineering and Computing 1983; 21: 566-72.

9 Hoffman P. An operation for severe grades of contracted or clawed toes. American fournal of Orthopedic Surgery 1912; 9: 441.

10 Fowler A W. A method of forefoot reconstruction. $\mathcal{F}$ Bone foint Surg [Br] 1959; 41: 507-13.

11 Kates A, Kessel L, Kay A. Arthroplasty of the forefoot. 7 Bone foint Surg [Br] 1967; 49: 552-7.

12 Jahss $M$ H. Spontaneous rupture of tibialis posterior tendon: clinical findings, tomographic studies, and a new technique of repair. Foot Ankle 1982; 3: 158-66.

13 Craxford A D, Stevens J, Park C. Management of the deformed rheumatoid forefoot. Clin Orthop 1982; 166:

14 Betts R P, Stockley, I, Getty C J M, Rowley D I, Duckworth $T$, Franks C I. Foot pressure studies in the assessment of forefoot arthroplasty in the rheumatoid foot. Foot Ankle 1988; 8: 315-26.

*PPT; Langer Biomechanica Group, Deer Park New York. †Spenco; Spenco Medical Corp, 6301 Imperial Drive, Waco, Texas.
Gordon E J, Ardizzone J. Clinical experiences with the SACH foot prosthesis. F Bone foint Surg [Am] 1960; 42: 226-34.

16 Newman R J, Fitton J M. Conservation of metatarsal heads in surgery of rheumatoid arthritis. Acta Orthop Scand 1983; 